\title{
ACERCAMIENTOS TEORICO-ÉTICOS SOBRE LA RELACIÓN ENTRE LA TERAPIA OCUPACIONAL Y LA CIENCIA: IMPLICANCIAS EN LAS PRÁCTICAS DISCIPLINARES
}

\author{
THEORETICAL APPROACHES ON THE RELATIONSHIP BETWEEN SCIENCE AND \\ OCCUPATIONAL THERAPY: IMPLICATIONS ON DISCIPLINARY PRACTICES
}

\section{Cristián Valderrama Núñez}

\begin{abstract}
Resumen
Este ensayo pretende reflexionar en torno a la relación entre algunos fundamentos de la ciencia y las implicancias en la práctica de la terapia ocupacional.

Este trabajo se aproxima a develar ciertos argumentos del saber científico que pueden ser cuestionados en sus implicancias cuando son aplicados en los sujetos o comunidades, específicamente el caso del inductivismo y la observación. Este ejercicio se realiza posicionando a la terapia ocupacional como un dispositivo, por tal motivo ésta es una práctica, un discurso, un saber que produce subjetivación, por ende las acciones de la terapia ocupacional no solo implican un posicionamiento epistemológico, sino que también ético y político.
\end{abstract}

Se desarrolla una alternativa al modelo médico de intervención, proponiendo la necesidad de incorporar un modelo social crítico de intervención en Terapia Ocupacional, argumentando los aspectos teóricos, éticos y políticos implicados en esta perspectiva.

Por último, se abre una ventana atrevidamente para repensar la perspectiva de desarrollar las ciencias de la ocupación desde una mirada sociocrítica.

\section{Palabras clave:}

Saber científico, ciencia social crítica, modelo social, diferencia.

\begin{abstract}
This essay aims to reflect on the relationship between some fundations of the science and implications for occupational therapy practice.

This work attempts to reveal certain arguments of scientific knowledge that can be questioned in its implications when applied to individuals or communities, specifically the case of inductivism and observation. This exercise is done by positioning occupational therapy as a device, for this reason, this is a practice, a speech, and a knowledge that produces subjectivity. Thus, the actions of occupational therapy involve not only an epistemological, but also an ethical and political positioning.
\end{abstract}

An alternative to the medical model of intervention is developed, suggesting the need to incorporate a critical social model of intervention in occupational therapy, arguing the theoretical, ethical and political issues involved in this perspective.

Finally, a window is boldly opened to rethink the perpective of developing occupational science from a sociocritical view.

\section{Keywords}

Scientific knowledge, critical social science, social model, difference

\footnotetext{
${ }^{1}$ Terapeuta ocupacional. Licenciado en ciencias de la ocupación humana U. de Chile. Magíster (c) en Terapia Ocupacional UNAB. Director de carrera Terapia Ocupacional UNAB sede Gran Concepción. 041-2662055 - 99597871. cristianto@gmail.com
} 


\section{INTRODUCCIÓN}

Las ciencias de la ocupación se encuentran en un momento histórico de desarrollo y profundas reflexiones. Motiva e impulsa el desarrollo de este ensayo, reflexionar sobre algunos argumentos que han sustentado la ciencia, tanto como forma de conocimiento y saber, así como fuente que determina las prácticas interventivas en aquellas disciplinas como la Terapia Ocupacional $(\mathrm{TO})^{2}$, que sustentan su hacer, en ese saber científico.

El propósito es aportar a una reflexión crítica sobre esos argumentos, de tal forma de develar las implicancias éticas y políticas que tienen sobre los sujetos que la disciplina interviene. Se aborda una contrapropuesta epistémica, de carácter sociocrítico, y por último, se discuten temas que emergen de esta contraposición, como la rigurosidad científica deshumanizante con el rescate del sujeto, la cosificación del sujeto en la intervención con la práctica disciplinar liberadora del sujeto, entre otros.

El método con el cual se lleva a cabo este ejercicio ensayístico, es una revisión bibliográfica. Se analizarán los siguientes ejes temáticos: la inducción y la observación como bases sobre las cuales se construye el saber y conocimiento científico, las diversas expresiones en la intervención a partir de estos saberes en problemas de salud mental de los sujetos, las implicancias éticas de operar a partir de estos supuestos, tanto en la generación del saber como en las prácticas de la disciplina. Y por último, aportar a la discusión de un enfoque de intervención social que rescata la subjetividad y los elementos sociales en la intervención.

2 Para efectos de este ensayo, la TO se considera un dispositivo. Moro (2006) señala tres aspectos fundamentales para entender la conceptualización de dispositivo, desde una perspectiva foucaulteana: primero, el dispositivo remite a una interacción de un conjunto heterogéneo de elementos (discursos, prácticas, normas, conceptos, leyes, instituciones, literatura, entre otros). Dicha relación es histórica y se ha modificado a través del tiempo; segundo, el dispositivo permite develar las relaciones de poder ocultas bajo la constitución de un campo del saber, el dispositivo es aquel espacio donde se entremezclan y cruzan los discursos cargados de verdad y los mecanismos, prácticas e instituciones de poder; en tercer lugar el dispositivo define procesos de subjetivación ligados a la constitución de un saber y al ejercicio de algunas relaciones de poder.

\section{La inducción, análisis y crítica}

Las ciencias se caracterizan principalmente por construirse a partir de dos tipos fundamentales de razonamientos y lógicas del pensar científico: el método hipotético deductivo y el método inductivo. En este ensayo abordaremos este último

La inducción consiste en inferir una conclusión o enunciado universal a contar de hechos, situaciones o enunciados particulares o singulares.

De esta forma se asume el cumplimiento de dos cualidades que garantizan la verdad: son universales, es decir, que lo que se afirma que ocurre, ocurrirá en todos los casos, y por otro lado, son necesarias, es decir, lo que se afirma que ocurrirá no ocurrirá de otra manera (Pérez, 2008).

Esto es particularmente relevante, cuando se considera la aplicación de este argumento en los seres humanos. Los sujetos forman parte de una colección o muestra infinita, por lo tanto $\mathrm{NO}$ es posible observar todos los casos, se desprende entonces que no se pueden realizar conclusiones universales y tampoco se puede afirmar que el próximo evento de la serie ocurrirá necesariamente igual al precedente. De este supuesto de la inducción, es cuestionable la aplicación de ciertos modelos de intervención o técnicas que garantizarían la recuperación en el ámbito de la salud mental de las personas, como por ejemplo, lo que plantean las corrientes conductistas ${ }^{3}$ (cuyas bases en el desarrollo de sus fundamentos se encuentran en los procesos de inducción), que es posible eliminar conductas desajustadas con técnicas como la extinción, cuyo ejemplo clásico es el "no responder a las pataletas" de los niños, con el supuesto de que así no se refuerza y se acabará la conducta desajustada del niño ${ }^{4}$.

Sin embargo, un elemento que permite considerar favorablemente las conclusiones universales es que aumentan la confianza para aplicar algunos modelos o estrategias y así mejorar la eficacia en la Intervención de TO.

\footnotetext{
3 Para Watson (1913), referenciado en Barlow y Durand (2003), "la psicología, desde el punto de vista conductista, es una rama experimental puramente objetiva de las ciencias naturales. Su objetivo teórico es la predicción y el control de la conducta. La introspección no forma parte de sus propósitos" (p. 26).

4 Este ejemplo, a mi parecer, instala que esta técnica (extinción) ignora elementos motivacionales y cognitivos de los niños o adultos que subyacen la complejidad del ser humano y que nos configura como seres únicos, y un conjunto infinito con una multiplicidad de diferencias y variantes subjetivas, difíciles de estandarizar por medio de técnicas.
} 
La confianza, como señala Pérez (2008) surge, ya que el método inductivo por sí mismo no logra alcanzar la verdad, pero sí permite acercarnos a ella todo lo que se desea, ese acercamiento es la confianza.

Por tanto, la eficacia implica que frente a un problema psicosocial o de salud mental, aplicar técnicas o métodos confiables, que están diseñados para abordar de una determinada manera un problema definido y no otro, nos permite tomar ciertas precauciones al advertir sobre prácticas no beneficiosas.

En otras palabras, nos sirven para "no dar palos a ciegas $^{\prime \prime}$ en la atención de los seres humanos. Por ejemplo, en la intervención psicosocial de la depresión: "la consejería, la intervención psicosocial grupal y la psicoeducación son recomendables para todas las personas con depresión, tanto en adolescentes como en adultos" (MINSAL 2009, p. 6). En el mismo documento, se recomienda en la depresión durante el embarazo o post parto, iniciar tratamiento con opciones terapéuticas no farmacológicas (intervenciones ambientales y psicoterapia).

\subsection{Las probabilidades como aliadas al inductivismo}

El concepto de inducción adquiere distintas expresiones, en este sentido la inducción probabilística corresponde a lo siguiente: «Si en una amplia variedad de condiciones se ha observado un gran número de $\mathrm{A}$ y si todos estos $\mathrm{A}$ observados poseen sin excepción la propiedad B, entonces probablemente todos los A poseen la propiedad B» (Chalmers 1992). Este principio surge de la necesidad de validar y moderar el principio de inducción. Sin embargo, a través de la lógica es posible fundamentar lo errado del argumento usado en este principio inductivo probabilístico. Esto es posible demostrarlo cuando se explican situaciones usando el argumento condicional, en el cual existen 2 premisas y una conclusión, el inductivismo propone que si dos premisas son verdaderas, la conclusión también lo será, sin embargo, el siguiente ejemplo demuestra que este razonamiento es erróneo:

\footnotetext{
5 Nota: Sin duda, que esto tiene un impacto favorable en el uso razonable y juicioso de las decisiones interventivas, destinadas a resolver problemas de salud mental, por supuesto sin negar la posibilidad de que en este camino de la intervención surjan métodos y estrategias caso a caso que sean pertinentes y significativas.
}

1. Muchos libros de psicología son aburridos.

2. Este libro es un libro de psicología.

3. Este libro es aburrido.

Las probabilidades se expresan en porcentajes; este dato es relevante para la explicación siguiente. Según lo planteado por Silva (2003) en los cuadros de deprivación moderada de alcohol, a pesar de las medidas terapéuticas básicas (régimen liviano, líquidos en abundancia, cuidados de enfermería y fármacos), se espera que alrededor del $5 \%$ de los pacientes evolucionen a un síndrome de deprivación severo que ocurre entre 1 a 4 días de la ingestión intensa de alcohol.

De este ejemplo, los datos no pueden ser generalizados, ya que no se puede incluir en esta conclusión al siguiente caso de deprivación moderada de alcohol que aparezca, por lo tanto, el siguiente caso de deprivación moderada de alcohol sometido a las medidas terapéuticas mencionadas tiene un $50 \%$ de probabilidades de evolucionar a un cuadro de deprivación severa y un $50 \%$ de no ocurrir así. Es más, podría ser que un sujeto que cursa un cuadro de deprivación moderada sin ningún tipo de tratamiento no evolucione a un cuadro de deprivación severa, incluso podría ser que un sujeto con tratamiento que cursa un cuadro de deprivación moderada evolucione a un cuadro de deprivación severa en 10 días.

El caso anterior permite cuestionar la supuesta capacidad predictiva de los procesos inductivos, situación muy usada en el ámbito de la medicina y especialmente la psiquiatría -campo tradicional e histórico de intervención de la TO-, por ejemplo, cuando se establece que "exponer a los niños al alcohol a una edad temprana en un escenario familiar o religioso" (Sarason y Sarason 2006, p. 456) es una de las condiciones socioculturales que reduce al mínimo los problemas del alcoholismo.

\subsection{Implicancias en la Terapia Ocupacional}

Se profundizará esta situación y complementará las críticas con un nuevo ejemplo:

Premisa 1: En ocasiones las personas que sufren de esquizofrenia manifiestan un afecto inapropiado

Premisa 2: esta persona padece esquizofrenia

Conclusión: esta persona manifiesta un afecto inapropiado. 
Es muy difícil determinar ciertos comportamientos tomando como base algunos hechos relativos sobre las personas, que además serán definidos en un determinado contexto de tiempo y espacio. En el caso recién mencionado, la conclusión puede ser errada, ya que una persona por el hecho de padecer esquizofrenia no necesariamente presenta un afecto inapropiado.

Desde la TO, esta conclusión sería catastrófica en términos de la Intervención a realizar, ya que estaríamos homogeneizando y estigmatizando a todas las personas que sufren esquizofrenia. Bajo esta lógica, implicaría asumir ciertas cadenas de consecuencias, por ejemplo, que el afecto inapropiado determinará en el sujeto afectado de esquizofrenia un desempeño ocupacional inadecuado.

Esto es de vital importancia para la terapia ocupacional, ya que estos datos deben ser usados como información general, que no provoquen el apropiarse de ciertos saberes con respaldo científico, ya que los Terapeutas Ocupacionales debemos ser capaces de entender y analizar a y con los sujetos que interactuamos e intervenimos, desde las siguientes dimensiones, las cuales estarían configurando el desempeño de la ocupación en los sujetos (Paganizzi, 2007).

- Dimensión personal - individual, en la cual se encuentran los aspectos de la subjetividad, resonancia singular de los hechos históricos, biográficos y biológicos de un sujeto dado.

- Dimensión grupal - convivencial, implica la interpretación de los otros del grupo convivencial le otorguen a aquellas realizaciones que hacen a la coexistencia cotidiana. Coexistencia en el marco institucional, familiar, entre otros.

- Dimensión cultural - social, reglas sociales, convenciones, leyes que rigen una cultura dada, con un franco carácter simbólico.

Esto significa que los sujetos que sufren esquizofrenia manifiestan un afecto inapropiado es simplemente un dato que no puede definir el marco de acción interventiva de la disciplina, sin embargo, es útil para iniciar una serie de investigaciones, averiguaciones o evaluaciones que permitan efectivamente considerar que la alteración de los afectos de las personas con esquizofrenia interfieren, y en qué medida, o no interfieren un desempeño ocupacional óptimo, considerando por su- puesto las dimensiones recién mencionadas en el análisis de la posible alteración de un afecto inapropiado.

Los procedimientos inductivos en esencia para el desarrollo de la $\mathrm{TO}^{6}$ aparentemente solo entregan información útil, a considerar, a tener en cuenta, sin embargo, es una información cuestionable; es más, es información solamente cuando permita corroborar datos con situaciones experimentadas en la vivencia y la práctica de los sujetos de intervención.

\section{La observación en la ciencia}

"la ciencia comienza con la observación; la observación proporciona una base segura sobre la que se puede construir el conocimiento científico, y el conocimiento científico se deriva, mediante la inducción de los enunciados observacionales" (Chalmers A. 1990. Pág. 23).

Esto implica que existe un sujeto, que observa por medio de los órganos de los sentidos (visión, audición, olfato, gusto o tacto) un objeto, situación o hecho externo, el cual será correctamente "conocido", ya que las percepciones son suficiente aval para ser utilizado como instrumento de descubrir la verdadera esencia de lo observado. Son muchos los ejemplos que se pueden mencionar en que la observación ha sido un elemento fundamental para descubrir algunas situaciones o hechos elementales que permitieron el desarrollo de algún conocimiento. Existen algunos ejemplos en el desarrollo del conocimiento científico que han comenzado por la capacidad de observación, especialmente de aquellos individuos capaces de percibir la profunda y significativa belleza que se oculta en las cosas cotidianas. Robert y Michelle Root-Bernstein (2002) nos entregan algunos de ellos:

\footnotetext{
6 Se propone iniciar la comprensión de la TO, como una disciplina que construya y reconstruya sus fundamentos epistemológicos y metodológicos en las ciencias sociales, ya que éstas permitirían argumentar y comprender la ocupación como un fenómeno social. Cuando señalo ciencias sociales, me refiero a las ciencias sociales críticas; para ello se cita lo planteado por Villalobos (2010), quien asume una posición marxista en la comprensión de la crítica: "la crítica tiene como objeto, primaria y autónomamente, la cancelación de las representaciones y teorías erróneas acerca del mundo. Su propósito y status es por tanto estrictamente cognoscitivo" (p. 58); lo crítico desde esta perspectiva implica que simultáneamente corresponde a un ejercicio cognitivo, político y ético.
} 
¿Cuántas veces nos hemos metido a la bañera sin darnos cuenta siquiera del aumento en el nivel del agua?, precisamente esto fue lo que permitió a Arquímedes comenzar a establecer la relación existente entre el volumen de agua desplazada y la densidad de los objetos. ¿Cuántas veces se ha contemplado el cielo y nos hemos preguntado por qué es azul?, esta duda generada de la observación fue la que llevó al físico John Tyndall a descubrir que el color del cielo depende de la difusión de la luz causada por el polvo y otras partículas (p.61).

En los ejemplos recién mencionados, se establece que la observación es útil para descubrir algunas situaciones puntuales, específicas y únicas tanto si analizamos las características personales del sujeto observador (habilidades auditivas, visuales o gustativas), los contextos socio-históricos y los objetivos particulares del uso de la observación para cada caso. Sin embargo, lo que es rescatable del uso de la observación, es que de alguna forma u otra es el nexo inicial entre el objeto, sujeto, situación, hecho o "realidad" observada y las percepciones del sujeto observador que se transformarán en juicios, formas de intervenir, ideas o hipótesis.

Es difícil imaginar otro medio de percibir y registrar la información entregada por nuestro entorno, si no es por medio de la percepción de los órganos de los sentidos, sea directa o indirectamente. El problema que plantea el proceso de inducción en relación a la observación, en el caso de los fenómenos sociales, es que debe ser utilizado en función de objetivos estimados razonablemente como favorables para los sujetos en relación a la construcción del conocimiento sobre ellos mismos.

\subsection{Las percepciones como un lugar controversial}

Parece ser imposible percibir la esencia de los objetos o situaciones observadas y observarlas objetivamente. Esto permite introducir la segunda crítica a la inducción que desarrollaré, a partir del siguiente planteamiento: "La objetividad de la ciencia inductivista se deriva del hecho de que tanto la observación como el razonamiento inductivo son objetivos en sí mismos, cualquier observador que haga un uso normal de sus sentidos puede averiguar enunciados observacionales" (Chalmers 1990, pág. 27).

Los seres humanos tenemos sensaciones, percepciones y representaciones. Las sensaciones ocurren cuando un estímulo actúa sobre un órgano sensorial llamado receptor del sistema nervioso, que es capaz de provocar una señal de transmisión hacia un centro integrador, que en este caso es el cerebro. Las percepciones son el acto de toma de conocimientos de datos sensoriales del mundo que nos rodea, es lo que Jaspers Ilamó "conciencia de objeto". Por último, las representaciones. son imágenes surgidas en la conciencia, reconocidas como un producto de sí mismo, son íntimas, dependen de la actividad psíquica y de la voluntad. Las representaciones corresponden a algo anteriormente percibido, Ilamadas mnémicas, o a algo inventado, conocidas como de la fantasía (Capponi, 2006).

La percepción es muy frágil de sufrir distorsiones, algunos trastornos de la percepción que están asociados a condicionantes propias de los sujetos observadores (cognitivas, afectivas y de conciencia), con lo que se explica la dificultad de garantizar la pureza del sujeto que observa un fenómeno. Las ilusiones son PERCEPCIONES FALSEADAS O DISTORSIONADAS DE UN OBJETO REAL. Jaspers las clasificó en: Por Inatención: el debilitamiento de la atención dificulta la captación nítida de las cosas y favorece el error perceptivo (cuando leemos una palabra que no estaba impresa en el texto). Catatímicas, pese a que la captación sensorial es correcta, hay un compromiso afectivo que predispone una falsa percepción (en estados de éxtasis se puede ver que las imágenes religiosas sonríen, en estado de miedo al mirar en la oscuridad a través de la ventana y el movimiento del árbol, confundirlo con una persona). Oníricas: en las que el compromiso de conciencia altera y distorsiona la percepción de los objetos (Caponni, 2006).

\subsection{La observación y la Terapia Ocupacional}

Como señala Paganizzi (2007), en cualquier ámbito de desempeño que se desarrolle la TO, es la observación cuidadosa la base de la evaluación y tratamiento, el desempeño profesional requiere desarrollar la capacidad de observar y oír.

En el ejercicio de la disciplina, en la relación permanente con otros sujetos, es fundamental observar con todos los órganos de los sentidos. Esto es de vital relevancia en cuanto en este encuentro con el otro, el Terapeuta Ocupacional sea capaz de identificar (evaluar, 
diagnosticar) y fomentar motivación (tratar, rehabilitar) en ocupaciones significativas y liberadoras para nuestros usuarios, ya que no contamos con instrumentos sofisticados ni tecnológicos para desarrollar una interacción y relación interpersonal con otros sujetos, sino que el uso de nosotros mismos como seres humanos con nuestras percepciones, experiencias e historia ${ }^{7}$.

Para la TO, es fundamental considerar que el sujeto interventor forma parte de una relación, en la cual incluye su propia subjetividad (cognición, afectos, creencias, valores, conciencia o inconsciencia) que son elementos enriquecedores y/o perturbadores en el ejercicio de la práctica, y como tales reconocernos y reconocer al otro para la construcción de saberes conjuntos y dialécticos con nuestros usuarios. Esto implica que no trabajamos con objetos observados, sino con sujetos activos, como lo plantean los principios del Modelo Canadiense del desempeño ocupacional, que refiere lo siguiente: "las personas de forma innata tienen la capacidad de elegir, organizar y desarrollar de forma satisfactoria ocupaciones significativas y culturalmente adaptadas, en búsqueda del cuidado personal, del disfrute de la vida y para contribuir a la sociedad" (Simó 2005, p. 11).

La observación, entendida como un instrumento, es para la disciplina, el rol que desempeña el terapeuta ocupacional como interventor, un sujeto con sentidos, percepciones, vivencias, emociones, pero por sobre todo un sujeto situado históricamente, que se enfrenta a contradicciones; que puede reproducir las condiciones sociales de opresión, desigualdad e injusticia social, o ser un facilitador de los procesos de cambios de los sujetos con los cuales trabajamos, un colaborador en la transformación social de los sujetos en situación de discapacidad ${ }^{8}$.

Nota del autor: El tema entonces es que la observación científica es un elemento más en una relación terapéutica compleja, donde hay dos o más sujetos activos con sus propias percepciones, historias de vida y emociones y sin duda alguna, solo observando no se logrará conocer la verdadera esencia de esos sujetos. Por lo tanto, el objetivo al observar estará en función de ser un elemento a considerar para generar vínculos, seleccionar qué proponer, facilitar experiencias significativas y dar inicio a relaciones sociales y ocupacionales más complejas.

8 Nota: Estos son los efectos en las subjetividades que puede producir la disciplina en el encuentro, práctica y convivencia del cuerpo disciplinar (los Terapeutas Ocupacionales) en el ejercicio de la intervención con los sujetos de atención.
Lo anterior implica reflexionar en torno a las maneras de intervención que se han desarrollado y pueden desarrollarse en la disciplina. El ejercicio de abordar las distintas maneras de Intervención, es para desarrollar en qué medida los principios de inducción y la observación se encuentran presentes en el Modelo Médico científico-natural, utilizado en algunas prácticas interventivas de Terapia Ocupacional. Posteriormente, se desarrolla una propuesta de un Modelo social de Intervención en Terapia Ocupacional, cuyos fundamentos se acercan a una ciencia social crítica.

\section{El Modelo Médico: expresión del saber cientí- fico natural}

Rafael Parada en Kottow, Bustos (2005), plantean que la medicina "entiende al enfermo como un objeto a estudiar..." (p. 50), "la medicina se define por su preocupación hacia el hombre enfermo..." (p. 51), "... La medicina ha comenzado a buscar las causas del enfermar y a desarrollar un pensamiento causal-determinista que continúa apegado a la medicina científico-natural" (p. 52).

A partir de estas características generales es posible interpretar que el Modelo médico produce los siguientes efectos: invisibiliza y fragmenta a los sujetos, cosificándolos para su propio conocimiento; este saber se centra en un estado del sujeto, estar enfermo, y no en su propia subjetividad y biografía. Se reduce la complejidad de la vivencia del sujeto enfermo a un cuerpo dolido.

Por otro lado, las estrategias interventivas se basan en que las enfermedades, como alteraciones orgánicas, requieren ser curadas, y esta curación se conoce como terapia.

\subsection{Implicancias en la Terapia Ocupacional, breve crítica a la apropiación disciplinar del saber médico científico natural}

Este modelo ha influenciado la Terapia Ocupacional en los procesos de evaluación e intervención. En la formación profesional se incluye fuertemente el aprendizaje de las enfermedades psiquiátricas o psicológicas que alteran el comportamiento ${ }^{9}$.

\footnotetext{
Nota: Este dato no es menor, porque es posible considerar que el solo hecho de enfocar un proceso de aprendizaje a la enfermedad implica establecer una mirada biológica, reduccionista de los sujetos, ya que en el estudio de éstas se encuentra el foco en
} 
Esto se refleja en la historia de la TO, cuando el psiquiatra William Rush, instruía que las actividades a realizar por los enfermos psiquiátricos debían estar asociadas a la disminución de los comportamientos inadecuados de cada patología. Es así que, las personas que sufrían depresión debían realizar actividades excitantes, los que padecían de manía debían realizar actividades relajantes, tranquilas, los que padecían de esquizofrenia debían desarrollar actividades que incluyan la interacción social, que fueran sencillas y estructuradas. ${ }^{10}$

En la propuesta anterior, se aplica la inducción en el sentido de que los sujetos diagnosticados con los cuadros psiquiátricos recién mencionados, deberían ser tratados en TO con la aplicación del mismo tipo de actividades para todos los casos incluidos en ese diagnóstico. La observación está implícita en la base con la cual se determina la categoría diagnóstica a la cual pertenecen los sujetos, es decir, a través de la observación de manifestaciones conductuales anormales que ellos presentan. ${ }^{11}$

la alteración, en la enfermedad. Esto es verificable al revisar mallas curriculares de diversas escuelas de Terapia Ocupacional, en ellas se encontrarán asignaturas como neurología, psiquiatría, reumatología, entre otras, y si se profundiza en quienes son los docentes en la mayoría probablemente encontraremos que son profesionales médicos especialistas en el área.

10 Nota del autor: Es fundamental considerar que es probable que estas prácticas no se encuentran extinguidas en algunos centros de atención psiquiátrica, donde se desarrolla la Terapia Ocupacional; ahí se puede considerar ser precavidos para evitar exacerbación de los síntomas, los cuales desde esta lógica limitarían la autonomía y realización personal de los usuarios.

11 Nota del autor: Lo más complejo de la influencia del modelo médico en la Terapia Ocupacional del ejemplo anterior, radica en que la ocupación también es reducida a actividades analizadas en función de paliar un síntoma como lo hace el modelo médico. Situación que transforma el ejercicio de la terapia ocupacional en la prescripción de recetas de actividades, así como el médico prescribe recetas de fármacos para los síntomas de los usuarios desde el modelo médico de alteración. Por tal razón sugiero que es importante conocer los cuadros clínicos (causas, mecanismos y efectos) porque nos permite establecer un diálogo y discurso con la disciplina imperante en los establecimientos de salud, pero que no puede definir nuestra práctica profesional por el solo hecho de pertenecer a un sistema establecido y legitimado por un tipo de saber específico. Por otro lado, es fundamental develar estas implicancias para considerar la posición político-ética de continuar reproduciendo estas prácticas o comenzar a modificarlas.
Planteados los argumentos necesarios para repensar los fundamentos teóricos y prácticos de la TO a partir del modelo médico, es necesario abordar el Modelo Social y sus modalidades para profundizar la propuesta de este trabajo y seguir repensando la disciplina.

\subsection{El modelo social como desviación, una expre- sión de la perspectiva médica}

Existen dos alusiones a la palabra norma; la primera es la Norma Ideal, que supone un cierto arquetipo perfecto del ser humano y ve degradaciones en todas las anormalidades en cuanto signifiquen un alejamiento de ese modelo. Es la norma del deber. Por ejemplo, los padres deben proteger a sus hijos, los niños deben estudiar, no casarse. Y la segunda es la Norma Estadística, en la cual lo normal es aquello que se observa más a menudo. La diferencia entre normal y anormal es cuantitativa. Las personas que sufren trastornos psiquiátricos serían anormales, al igual que las personas con un Coeficiente Intelectual superior (Caponni, 2006).

Pérez (2012) propone una interpretación de la alteración, que comprende a ésta como una anormalidad en el orden de lo social; cuando esto ocurre se considera una desviación. Las conductas extravagantes o alteraciones del comportamiento corresponden a esta categoría. Las desviaciones "son consideradas desde sus manifestaciones...", "...un comportamiento desviado está precedido por criterios fuertemente valóricos, y sometida a toda clase de tensiones sociales, contextuales e históricas...", "...en las desviaciones sociales no es fácil distinguir entre causa y efecto, y ambos interactúan fuertemente entre sí." (p. 211).

Ya que las desviaciones sociales como la locura "representan verdaderos desafíos a la normalidad social en el plano directamente valórico y político" (Pérez 2012, p. 215), esta situación se debe corregir, y la corrección está enfocada a adaptar al sujeto desviado a las normas establecidas, prepararlo para integrarse a lo que la mayoría de las personas de su edad, sexo, condición social u otra variable dominante determine hacer. Es decir, disciplinar; a través del disciplinamiento se normaliza la desviación social.

Esto define que el sujeto no tiene una influencia relevante en el proceso de su propio bienestar social, es decir, se adapta o se adapta, no hay espacios para la diferencia, en este adaptarse lo que ocurre con el sujeto es que es sometido a un disciplinamiento terapéutico, 
esta forma de concebir la alteración corresponde a un enfoque funcionalista de la sociedad, el cual Galheigos (2007) describe de la siguiente manera:

\begin{abstract}
Supone que la sociedad es una organización sistémica y funciona según unas bases consensuadas; la marginalidad ocurre cuando uno de sus miembros no consigue adaptarse a estas reglas. La marginalidad y la falta de integración se reducen al éxito o fracaso de la adaptación de una persona a una estructura social determinada (p. 87).
\end{abstract}

La influencia de esta mirada de la alteración en la TO es que la mayor parte del desarrollo teórico del dispositivo disciplinar considera como base esta perspectiva de la alteración, esto ha significado considerar al sujeto como un depositario de habilidades y competencias, funcionales para la adaptación social. En la cotidianidad, el sujeto debe ser capaz de tolerar las exigencias y reglas de un ambiente social homogeneizado y demandante, en el cual "todos deben remar para el mismo lado's".

Esto finalmente se refleja, cuando se realizan programas terapéuticos, en los cuales se homogeneizan y estandarizan ciertas habilidades o capacidades, y se transforman a éstas en una condición individual necesaria para convivir socialmente, quedando la sensación de que la desviación debe notarse lo menos posible. En la disciplina TO esto se ha conocido como integrar, a través de la práctica interventiva se prepara al sujeto, considerando elementos subjetivos; sin embargo, esto

12 Nota: la frase tiene la intención de no obviar que muchas de estas exigencias surgen de los mecanismos propios del sistema neoliberal de producción económica, el cual ha transformado a los sujetos en consumidores/labradores (García de la Huerta, 2010), lo que implica que la generación de subjetividades depende de la relación del sujeto con el mercado, las relaciones de producción y las opciones reales de consumo. De ahí desprendo que la subjetividad implica el desarrollo de competitividad, emprendimiento, preparación intelectual y actitudinal, entre otras. Lo fundamental de este planteamiento es que esta condición implica la incorporación de todos, todos se impregnan de esta necesidad de pertenecer a este sistema. Por tanto, las personas en situación de discapacidad, los vulnerados sociales, también deben ser parte de esta maquinaria socioeconómica, pero para ser parte de él, deben ser normales, ser o aparentar ser como todos. Una lógica inductiva subyace esta práctica. implica lograr estados de desempeños óptimos, semejantes a lo establecido social y culturalmente para todos los sujetos. ${ }^{13}$

Otra expresión de prácticas del dispositivo disciplinar, que podría ser discutida por sus efectos éticos, es cuando los terapeutas ocupacionales dirigen programas de reinserción laboral "protegida", considerando que estas modalidades reemplazan un empleo, de tal forma que se consideran una forma de normalizar el desempeño ocupacional de los sujetos, corriendo el riesgo de promocionar la discriminación "positiva".

Con esto se propone que la TO debe ser cautelosa, porque es cuestionable que por medio de estas prácticas, con una supuesta base científica, en su quehacer la disciplina mantenga un sistema de desigualdades con los sujetos que sufren alguna desviación, especialmente cuando se comienzan a desarrollar discursos al interior de los dispositivos vinculados a fomentar la inclusión social, el fortalecimiento social, derribar las desigualdades, entre otros.

Sin embargo, una defensa de esta posición teórico -práctica (la desviación), es establecer que así está estructurado y funciona el sistema social, que las presiones sociales en la vida cotidiana son cada vez mayores, por lo tanto las personas deben contar con una base mínima de competencias para enfrentar la carga psicosocial a la cual se está sometido, de lo contrario, si la TO no incorpora al sistema de producción a los sujetos de atención, las oportunidades de autorrealización y bienestar personal estarán disminuidas, y simultáneamente podría aumentar la marginalidad de los sujetos que sufren alguna alteración del comportamiento o se encuentran en situación de discapacidad.

\footnotetext{
13 Nota: con esta lógica se desarrollan talleres prelaborales, esperando entrenar competencias como responsabilidad, puntualidad, manejo adecuado con la autoridad, empatía, asertividad y capacidad de resolver conflictos, etc., necesarias para desempeñar un empleo, como por ejemplo, el ser estafeta, jardinero o conserje, que dicho sea de paso, rara vez, en las mismas condiciones salariales cuando se trata de una persona en situación de discapacidad. O que los terapeutas ocupacionales insistamos en que las personas desinstitucionalizadas de hospitales psiquiátricos comiencen a ocupar espacios comunitarios como asistir al cine, a un teatro, a un mall, para realizar lo que todas las personas de su edad, y sexo realizan, es decir, incorporarse a la norma, ser consumidores.
} 


\subsection{El modelo social para la diferencia, una perspectiva crítica}

La segunda visión de la alteración desde el modelo social, corresponde a que la alteración sería una diferencia $^{14}$ (Pérez, 2012). Esto quiere decir que el hecho de sufrir una alteración es visto como una condición de los sujetos ${ }^{15}$, por tanto, no es una enfermedad que se pueda curar, ni una desviación que se deba corregir.

La diferencia para que sea tolerada y reconocida por el entramado social, requiere una sociedad tolerante, inclusiva de la realización personal de los sujetos diferentes. Esta es una dimensión para la TO extremadamente importante, fundamental para su quehacer; sería necesario incorporar un nuevo foco de intervención, la sociedad, el campo social, la justicia social y ocupacional.

Al mismo tiempo, es necesario comenzar a incorporar en el abordaje interventivo, consecuencias de las sociedades no inclusivas, represivas y discriminadoras, como el apartheid ocupacional, es decir, "las restricciones y negaciones de acceso a una participación digna y significativa en las ocupaciones de la vida diaria, basada en la raza, color, discapacidad, edad, sexo, orientación sexual, religión, u otra característica" (Kronemberg et al. 2007. Pág. 87).

El validar la mirada de la diferencia ante la alteración, es para la TO un elemento a considerar en las metodologías de intervención. En un estudio de Jorge Chuaqui (2005) quedan de manifiesto algunas conclusiones, en las que los empresarios estigmatizan los comportamientos y posibles rendimientos de los sujetos con esquizofrenia con una valoración negativa, situación generada desde la falta de conocimiento y el manejo negativo de los medios de comunicación y la sociedad de la locura. Sin embargo, la pregunta es: ¿Qué hace la

14 Nota: en la diferencia los sujetos son reconocidos como tales. Esta mirada de la alteración, está vinculada al concepto de inclusión, es decir, que la alteración en un medio social inclusivo no es rechazada, cuestionada o valorada negativamente, por el contrario es aceptada, es validada como una condición, como una cualidad dentro de un pool de cualidades que poseen los sujetos que componen y producen la sociedad. Los sujetos con capacidades distintas no deben adaptarse, no están fuera de la norma, son parte de un todo, existe un reconocimiento de éstos.

15 Es particularmente importante esta mirada para la TO, ya que propone cambiar el foco de intervención en los contextos sociales, en los significados de la alteración en los sujetos, quienes a partir de sus relaciones intersubjetivas van redefiniendo su sentido de identidad, es decir existe una política de la identidad. terapia ocupacional en ese ámbito?, ¿qué estrategias se han diseñado a la luz de los datos para que se transformen las sociedades, en grupos inclusivos y respetuosos de la diferencia?

El desafío está planteado y la disciplina debe asumirlo, ya no basta con atender y rehabilitar a los usuarios, la TO debe realizar esfuerzos para habilitar a la sociedad en su conjunto. ¿Qué pasaría si intervenimos a los empresarios?, ¿cuántos puestos de trabajo en condiciones adecuadas se lograrían garantizar a las personas diferentes? ¿En qué medida la TO se preocupa de las condiciones sociales de producción de la subjetividad de los sujetos que interviene? ¿Conocerán los sujetos no vinculados a la rehabilitación o a la inclusión social, qué implica la diferencia en lo cotidiano, en la praxis?

\subsection{Implicancias en las prácticas de la Terapia Ocupacional: la terapia, el disiciplinamiento y la Intervención como tal}

La terapia es un concepto que se aplica al referirnos a la alteración como una enfermedad desde el modelo médico. El concepto de disciplinamiento se aplica cuando hablamos de la alteración como un fenómeno social Ilamado desviación. El concepto de intervención es usado cuando nos referimos desde el modelo social a la alteración, siendo ésta entendida como una diferencia (Pérez, 2012).

La terapia y el disciplinamiento se caracterizan porque se desarrolla en la base de una relación vertical entre un sujeto (experto, que sabe) y un sujeto enfermo (que no sabe, que necesita a alguien que sabe). Esta situación supone que el sujeto no es quien conoce su realidad, sus representaciones de la realidad, ni sus formas de afrontamiento funcionales o disfuncionales, siendo el experto quien se las señala, da a conocer, y enseña a utilizarlas (Pérez, 2012).

La terapia ${ }^{16}$ y el disciplinamiento, al mismo tiempo aplica las técnicas, están previamente establecidas para cada problemática, por ejemplo, en las personas diagnosticadas con depresión la técnica cognitiva conduc-

\footnotetext{
16 Se usa el término en forma genérica, para enmarcar una serie de acciones curativas que tiene distintas expresiones en diversas disciplinas como la medicina, la psicología, y la propia TO, cuando en su acción terapéutica reproducen las características señaladas en la descripción.
} 
tual que recupera las distorsiones cognitivas que aparecen en este estado de ánimo son la reestructuración cognitiva: si la situación es superar un miedo patológico se usa la aproximación progresiva al estímulo desencadenante del miedo, si el caso es mejorar las habilidades sociales en una persona que padece esquizofrenia, la técnica es el juego de roles (Durand y Barlow, 2003). Entonces el experto debe ser capaz de definir con precisión el problema a tratar en la etapa de evaluación, para aplicar la técnica más acorde a esa problemática.

De esta idea, se desprende que la terapia y el disciplinamiento son de carácter individual, tienden a la homogeneización, es decir, se aplican técnicas y estrategias de la misma forma para todos. El experto adiestra y entrena destrezas para superar la alteración y lograr los estándares aceptados. El agente del cambio es el experto (Pérez, 2012).

Por su parte la intervención, establece varias diferencias con la terapia y el disciplinamiento. La intervención supone una relación horizontal (Pérez, 2012), en cuyo encuentro se comparten saberes, lo que podríamos decir es que dialogan el saber científico con el saber popular, para crear un nuevo saber y principalmente una nueva práctica. ${ }^{17}$

La intervención produce un efecto diferenciador de los sujetos tanto en el grupo participante como de los estándares externos dominantes; cada participante es parte de un todo, sin dejar de lado su individualidad, en esta individualidad se involucra en el grupo o comunidad, al cual modifica y del cual forma parte, así como la interacción social lo transforma. En este sentido se produce lo que señala Pérez, 2012: "una integración diferenciadora" (p. 224), lo que podríamos mencionar equivale a una sociedad inclusiva.

La intervención produce el fortalecimiento, la cohesión de los sujetos considerados diferentes, la auto-

\footnotetext{
17 Nota autor; la intervención en este caso desarrolla técnicas, estrategias caso a caso, no están predefinidas las herramientas interventivas, por ejemplo, si deseamos intervenir en personas mayores de 65 años que presentan como factor de riesgo el aislamiento social. $\mathrm{Si}$ las personas deciden reunirse una vez por semana, en los distintos hogares de los participantes, el facilitador, potencia y valida dicha decisión, lo hace tanto como un rescate del sujeto y la expresión de su subjetividad, que caería en el orden de lo ético-político, como un ejercicio argumentativo cognitivo de la intervención social de estos sujetos. Los interventores están comprometidos con el cambio social y trabajando conjuntamente con la diferencia para lograr procesos de concientización, que aproximen a la transformación de las condiciones materiales de existencia.
}

nomía y la autoorganización en función de validarse como sujetos de derecho.

\section{Conclusiones}

Cuando el principio de inducción y observación son considerados como fundamentos incuestionables o son naturalizados para la acción con seres humanos, es necesario que la disciplina sea precavida.

La rigurosidad en el pensar y actuar en los postulados de la ciencia implica en muchas ocasiones ignorar las intersubjetividades y subjetividades ${ }^{18}$. Sin duda que los argumentos científicos - naturales permiten resolver problemas reales y concretos de algunas problemáticas particulares de sujetos, existe una TO tradicional e histórica que mantiene en sus prácticas esos principios, lo cual es totalmente legítimo y válido. La intención no es evaluar negativamente esos saberes y prácticas, muy por el contrario, es proporcionar una discusión para fortalecer las perspectivas disciplinares, principalmente dirigidas a la función cognoscitiva, ética y política de ésta.

Para ello, es relevante rescatar al sujeto de intervención, colocar el foco de atención en las condiciones sociales de producción de las subjetividades, instalar la ocupación como un fenómeno social y no individual, repensar o formular una ciencia de la ocupación sociocrítica.

Un aporte para avanzar hacia ello, son los principios enunciados de la Intervención de la diferencia, ya que éstos corresponden a los argumentos teóricos, éticos y políticos orientadores de una práctica de la TO con una perspectiva sociocrítica; esta se propone como una nueva forma de pensar la práctica de la TO, especialmente para sostener la resolución de las problemáticas emergentes de la TO actual, como es la inclusión educacional, inclusión laboral, sujetos privados de libertad, sujetos en situación de calle, entre otros.

\footnotetext{
18 La intersubjetividad y la subjetividad son dos conceptos interdependientes uno de otro. Schutz plantea que la subjetividad no es posible sin la presencia de la intersubjetividad, es decir los significados subjetivos se construyen considerando al otro y en interacción con el otro, hecho que ocurre en el marco de la vida cotidiana. De estas formas la fuente que construye las subjetividades individuales es la intersubjetividad como interacción social de esas mismas subjetividades, por tanto las subjetividades construyen al mismo tiempo lo social, el campo de lo social, como una relación dialéctica (Hernández y Galindo, 2007).
} 
La TO eventualmente pudiese desarrollar los 3 conceptos: terapia, disciplinamiento e intervención, la decisión de ello es un asunto ético-político.

Si el sistema de salud, campo del cual procede la disciplina, usa la terapia, la TO con perspectiva social crítica podría tensionar la incorporación de nuevas prácticas centradas en la diferencia. La TO es un agente social y político responsable, que debe velar porque los métodos de abordaje se usen criteriosa y éticamente, en beneficio de los sujetos.

Procurar que no exista abuso de poder de la institucionalidad sobre los sujetos de intervención, como ocurre en algunos hogares protegidos, hogares de ancianos, hospitales psiquiátricos, "instituciones de salud", procurar no reproducir las normas de lo instituido si no benefician a los sujetos intervenidos, como en el uso de psicofármacos sin la información adecuada para que decidan si usarlos o no, de alguna forma el TO es el puente político y técnico entre lo normativo y el derecho, por lo que se debe estar consciente de los fines que busca cada medida.

En definitiva como terapeutas ocupacionales se puede aplicar la terapia, el disciplinamiento o la intervención siempre y cuando procuremos garantizar un respeto a los derechos y dignidad de nuestros usuarios, considerando a éstos como sujetos capaces de determinar por sí mismos su realización y autonomía personal, y para ello es necesario brindar las condiciones materiales para que ello ocurra.

\section{ReFERENCIAS BibLIOGRÁFICAS}

Barlow D., Durand M. (2003). Psicopatología. 3era edición. Editorial Thomson

Caponni R. (2006). Psicopatología y semiología psiquiátrica. 11a edición. Editorial Universitaria.

Chalmers A. (1990). ¿Qué es esa cosa llamada ciencia? Una valoración de la naturaleza y el estatuto de la ciencia y sus métodos. 11a edición. Siglo XXI editores.

Chuaqui J. (2005). El estigma en esquizofrenia, Revista electrónica de la Universidad de Viña del Mar. Ciencias Sociales online. Vol. II, No 1.

Galheigo, S. (2007). Terapia Ocupacional en el ámbito social. En F. Kronenberg, S. Simó y N. Pollard, Terapia Ocupacional Sin Fronteras: Aprendiendo del espíritu de supervivientes (pp. 85 97). Buenos Aires: Médica Panamericana.
García, M. (2010). Foucault y Neoliberalismo: una lectura crítica. En V. Lemm, Michel Foucault: Neoliberalismo y biopolítica. (p. 177 - 197). Santiago: Ediciones Diego Portales.

Hernández, Y. y Galindo, R. (2007). El concepto de intersubjetividad en Albert Schutz. Espacios Públicos, 10 (20), 228-240. Extraído el 20 de enero de 2012 desde http://redalyc.uaemex.mx/ pdf $/ 676 / 67602012 . p d f$

Kottow M., Bustos R. (2005). Antropología Médica. Santiago. Editorial Mediterráneo.

Ministerio de salud. Subsecretaría de salud pública. 2009. Guía Clínica tratamiento de personas con depresión. Serie Guías clínicas MINSAL. Stgo. Chile.

Moro, O. (2006). La perspectiva genealógica de la historia. Santander: Servicio de Publicaciones de la Universidad de Cantabria.

Paganizzi L. (2007). Terapia Ocupacional Psicosocial. Escenarios clínicos y comunitarios. 1era edición. Buenos Aires. Editorial Polemos.

Pérez C. (2008). Hacia un concepto histórico de ciencias. Santiago: LOM Ediciones.

Pérez C. (2012). Una nueva antipsiquiatría. Crítica y conocimiento de las técnicas de control psiquiátrico. 1era edición. Santiago. LOM Ediciones.

Sarason I., Sarason B. (2006). Psicopatología: psicología anormal. El problema de la conducta inadaptada. México; 11a edición. Pearson practice Hall.

Simò S. (2006. Febrero). El Modelo canadiense del desempeño ocupacional I. Revista gallega de Terapia Ocupacional. Número 3. Consultado en http://revistatog.3owl.com/num3.htm

Root-Bernstein Robert y Michelle (2002). El secreto de la creatividad. Primera edición. Editorial Kairos.

Kronemberg F., Simó S., Pollard N. (2007). Terapia Ocupacional sin Fronteras. Aprendiendo del espíritu de supervivientes. 1a edición. Editorial Médica Panamericana.

Silva Hernán et al. 2003. Manual de psicofarmacología Clínica. 1a edición. Editorial Mediterráneo.

Villalobos M, (2010). Ciencia Social como crítica social, la revolución epistemológica de Karl Marx. 1a edición. Santiago, LOM Ediciones. 